\title{
Radiation tolerant fiber Bragg gratings for high temperature monitoring at MGy dose levels
}

\author{
A. Morana, ${ }^{1,2, *}$ S. Girard, ${ }^{1}$ E. Marin, ${ }^{1}$ C. Marcandella, ${ }^{3}$ P. Paillet, ${ }^{3}$ J. Périsse,${ }^{4}$ J.-R. Macé,${ }^{5}$ \\ A. Boukenter, ${ }^{1}$ M. Cannas, ${ }^{2}$ and Y. Ouerdane ${ }^{1}$ \\ ${ }^{1}$ Laboratoire Hubert Curien, Université Jean Monnet, CNRS UMR 5516, 18 Rue Prof. B. Lauras, 42000 Saint-Etienne, France \\ ${ }^{2}$ Dipartimento di Fisica e Chimica, Università di Palermo, via Archirafi 36, 90123 Palermo, Italy \\ ${ }^{3}$ CEA, DAM, DIF, F91297 Arpajon, France \\ ${ }^{4}$ Areva NP, 10 rue Juliette Récamier, 69456 Lyon Cedex 06, France \\ ${ }^{5}$ Areva, Tour Areva, 1 place Jean Millier, 92084 Paris La défense Cedex, France \\ *Corresponding author: adriana.morana@univ-st-etienne.fr
}

Received July 14, 2014; revised August 9, 2014; accepted August 9, 2014;

posted August 11, 2014 (Doc. ID 216992); published September 5, 2014

\begin{abstract}
We report a method for fabricating fiber Bragg gratings (FBG) resistant to very severe environments mixing high radiation doses (up to $3 \mathrm{MGy}$ ) and high temperatures (up to $230^{\circ} \mathrm{C}$ ). Such FBGs have been written in two types of radiation resistant optical fibers (pure-silica and fluorine-doped cores) by exposures to a $800 \mathrm{~nm}$ femtosecond IR laser at power exceeding $500 \mathrm{~mW}$ and then subjected to a thermal annealing treatment of $15 \mathrm{~min}$ at $750^{\circ} \mathrm{C}$. Under radiation, our study reveals that the radiation induced Bragg wavelength shift (BWS) at a 3 MGy dose is strongly reduced compared to responses of FBGs written with nonoptimized conditions. The BWS remains lower than $10 \mathrm{pm}$ for temperatures of irradiation ranging from $25^{\circ} \mathrm{C}$ to $230^{\circ} \mathrm{C}$ without noticeable decrease of the FBG peak amplitude. For an applicative point of view, this radiation induced BWS corresponds to an additional error on the temperature measurements lower than $1.5^{\circ} \mathrm{C}$, opening the way to the development of radiation-tolerant multi-point temperature sensors for nuclear industry. (c) 2014 Optical Society of America

OCIS codes: (060.2370) Fiber optics sensors; (060.3735) Fiber Bragg gratings; (350.5610) Radiation; (160.6030) Silica. http://dx.doi.org/10.1364/OL.39.005313
\end{abstract}

Since the nuclear disaster at Fukushima Daiichi in 2011, the nuclear industries are in the process of increasing the safety of their facilities by implementing new security systems and sensors that can survive the increasing environmental constraints associated with accidental scenarios. Depending on the event, expected conditions can be very severe in terms of radiation (doses up to $\operatorname{MGy}\left(\mathrm{SiO}_{2}\right)$ level, temperatures up to $\left.200^{\circ} \mathrm{C}[\underline{1}]\right)$. This is the case, for example, of temperature sensors around spent fuel pools. For this aim, fiber-based sensors like those based on fiber Bragg gratings (FBGs) present several advantages, like a faster time response than thermocouples (lower than $1 \mathrm{~s}$ ) and a high radiation tolerance, as investigated by previous studies [2]. However, radiation is also known to alter the fiber transmission properties as well as the FBG properties [2,3]. Furthermore, the amplitudes and kinetics of these changes are dependent on temperature, as this extrinsic parameter governs the stability of the radiation induced point defects in the silica-based glass at the origin of these macroscopic changes [2,3]. Another important point, as clearly shown in a paper by Henschel et al. [4], is that the radiation sensitivity of a fiber and of an FBG written in this fiber are not directly correlated but will depend on many other parameters like the FBGs' writing technique and conditions as well as the possible hydrogen-loading before inscription [5].

In this Letter, we performed, for the first time to the best of our knowledge, a complete study on a large series of FBGs in well-known radiation tolerant optical fibers to determine the optimal conditions of elaboration of FBGs that can withstand both high doses and high temperatures. We demonstrated the resistance of the best FBG, mixing both high irradiation doses up to 3 MGy and temperatures of $230^{\circ} \mathrm{C}$. Such FBGs are really efficient temperature sensors thanks to the dependence on the temperature of their Bragg wavelength, defined as []

$$
\lambda_{B}=2 \cdot n_{\mathrm{eff}} \cdot \Lambda,
$$

where $n_{\text {eff }}$ is the effective refractive index of the propagating core mode and $\Lambda$ is the period of the refractive index modulation in the fiber core.

At the sensor level, the radiation induced attenuation (RIA) is very impacting when the dose increases, as RIA can strongly decrease the sensing length, resulting sometimes in a total loss of the information. To avoid this, specific fibers composition should be used, the most efficient ones being those with pure-silica or fluorinedoped cores [3]. Then, these two types of radiation resistant single-mode fibers are used in this Letter and will be functionalized with FBGs. These fiber types are not photosensitive to UV laser light, so the FBGs have to be written with other techniques, less based on the photosensitivity of silica glasses. Here, we used an ultrafast Ti:sapphire laser emitting $50 \mathrm{fs}$ long pulses at $800 \mathrm{~nm}$ with a repetition rate of $1 \mathrm{kHz}$, to write the FBGs through a phase mask. The ultrafast laser was focused on the optical fiber core with the help of a cylindrical lens having a focal length of $10 \mathrm{~mm}$. A scan of the beam along the whole fiber core was performed at a frequency of $25 \mathrm{mHz}$ to homogenize the induced refractive index by the technique reported by Mihailov et al. [7]. The laser power was adjusted between 400 and $600 \mathrm{~mW}$ as a function of the desired grating strength. Even if the fibers used in this study are not photosensitive, no hydrogenation pretreatment was performed to enhance the grating writing efficiency. A systematic study was performed with many FBG sets having different responses to both pre-irradiation thermal treatments and radiations. It is 
well known that during the thermal annealing treatments the grating amplitude is reduced and the gratings can even be erased [8]. However, it has been demonstrated that with increasing inscription laser power the thermal resistance of the gratings increases [9]. For our writing setup, a laser power higher than $500 \mathrm{~mW}$ was found to be the optimal condition to create FBGs that can withstand a temperature treatment up to $750^{\circ} \mathrm{C}$. In this Letter, we focused the characterization study on three FBGs written under the same laser conditions $(>500 \mathrm{~mW}$ ) in both pure-silica core (PSC) and F-doped fibers and then subjected to three different pre-thermal treatments: one grating was not thermally treated; while the second and the third were annealed at $350^{\circ} \mathrm{C}$ and $750^{\circ} \mathrm{C}$, respectively, for $15 \mathrm{~min}$ (heating ramp of $10^{\circ} \mathrm{C} / \mathrm{min}$ for both cases). To put in evidence the thermal treatment effects on the peak amplitude of the FBG, Fig. 1 shows the transmission spectra of a grating before and after the treatment at $750^{\circ} \mathrm{C}$. In this case the amplitude is reduced from about 20 to $2 \mathrm{~dB}$. The gratings were not recoated after the annealing.

The irradiation tests were performed by using two $10 \mathrm{keV}$ x-ray machines at CEA, Arpajon in France, at room temperature (RT), about $25^{\circ} \mathrm{C}$, with a dose rate of $50 \mathrm{~Gy}\left(\mathrm{SiO}_{2}\right) / \mathrm{s}$. To study the effects of a pre-irradiation, two successive runs with an accumulated dose of 1.5 MGy each were performed with durations of $3 \cdot 10^{4} \mathrm{~s}$. The experimental setup is shown in Fig. 2: two or three FBGs were fixed stress-free on the heating plate of the irradiation system and their transmission spectra were recorded in parallel by using a tunable laser source (Tunics Plus from NetTest) and the CT400 high performance optical tester from Yenista Optics, with a wavelength resolution of $1 \mathrm{pm}$.

The temperature was controlled by a highperformance Temptronic thermo-chuck and monitored by two thermocouples (of type $\mathrm{K}$ ) that were fixed on both sides of the gratings at a distance of about $1 \mathrm{~mm}$. As both gratings and thermocouples were placed on the heating plate, all the sensors record the same temperature changes during and after the irradiations. Before irradiation, we varied the temperature from $20^{\circ}$ to $40^{\circ} \mathrm{C}$, and we recorded in situ the Bragg wavelength shift (BWS) that is intrinsically temperature dependent, but it is also influenced by temperature-induced strain variations. By using the relation,

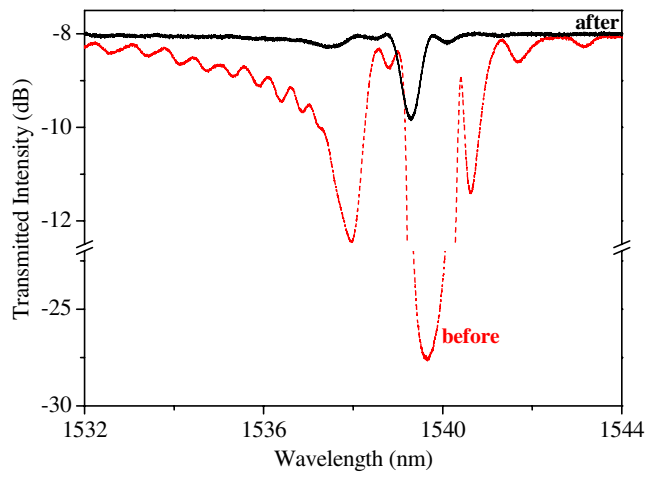

Fig. 1. Transmission spectra of a grating written in the PSC fiber, recorded at $21^{\circ} \mathrm{C}$ before (dashed line) and after (continuous line) the thermal treatment at $750^{\circ} \mathrm{C}$.

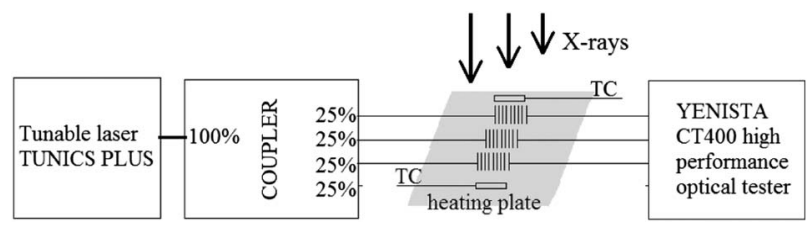

Fig. 2. Experimental setup used to record the transmission spectra of FBGs during irradiation. TC, thermocouple.

$$
\operatorname{BWS}(T)=\lambda_{B}(T)-\lambda_{B}\left(T_{0}\right)=\alpha \cdot\left(T-T_{0}\right),
$$

we determined the gratings coefficient $\alpha$. It is worth noting that the temperature dependence of FBGs is not linear over large ranges [6]; however the sensitivity can be considered constant over the range of $20^{\circ} \mathrm{C}$. This calibration allows us to compensate any temperature changes during irradiation (in our experiments it is lower than $4^{\circ} \mathrm{C}$ ) and therefore to calculate the radiationinduced peak shift. The best-fit value of $\alpha$ around $25^{\circ} \mathrm{C}$ varies between 9 and $15 \mathrm{pm} /{ }^{\circ} \mathrm{C}$ depending on the investigated FBGs. In particular, the FBG sensitivity decreases with increasing pre-thermal treatment temperature.

Two main effects are expected during the irradiations of FBGs; the first one is a shift of the Bragg wavelength caused by radiation and the second one can be a decrease of the FBG amplitude with the dose. We first report on the radiation-induced BWS that at the sensing level will be responsible for an increase of the temperature error. Then, we present the FBG amplitude changes with the dose, which do not affect the precision of the temperature measurements but will decrease the signal to noise ratio.

The Bragg wavelength position is determined by using a third order polynomial approximation of the peak (to account for a possible response asymmetry) on 200 points of the transmission spectrum near the main dip.

Figure 3 reports the radiation induced BWS at RT, corrected with respect to the small temperature fluctuations, of the FBGs written in the two fiber types and differently thermally treated. For both FBGs not thermally treated, $\lambda_{B}$ shifts toward shorter wavelengths during irradiations and toward the longer ones during recoveries: the first irradiation causes a larger shift for the F-doped fiber than for the PSC fiber (60 pm against $20 \mathrm{pm}$ at 1.5 MGy dose). Moreover, it is easy to note that a pre-irradiation increases the radiation resistance of the gratings, indeed further irradiations cause a BWS lower than $30 \mathrm{pm}$. For the thermally annealed gratings, the BWS behavior under radiation is inverted: the peak wavelength shifts toward longer values during irradiations and toward the shorter ones during recoveries. If the gratings are pretreated at $350^{\circ} \mathrm{C}$, the BWS initially increases with the dose; then it reaches a maximum value, that is smaller for the F-doped fiber than for the PSC one ( $8 \mathrm{pm}$ versus $27 \mathrm{pm}$ ), and it starts to decrease. As in the previous case, a preirradiation decreases the BWS, that is nearly $0 \mathrm{pm}$ for the first fiber and $9 \mathrm{pm}$ for the second one, after the second run. Finally, if the gratings are annealed at $750^{\circ} \mathrm{C}, \lambda_{B}$ increases up to only about $10 \mathrm{pm}$ at the total dose of $3 \mathrm{MGy}$, this corresponds to an error on the temperature measurement of about $1^{\circ} \mathrm{C}$. From these graphs it is clearly highlighted that a pre-thermal treatment increases 
(a) F-doped core fiber

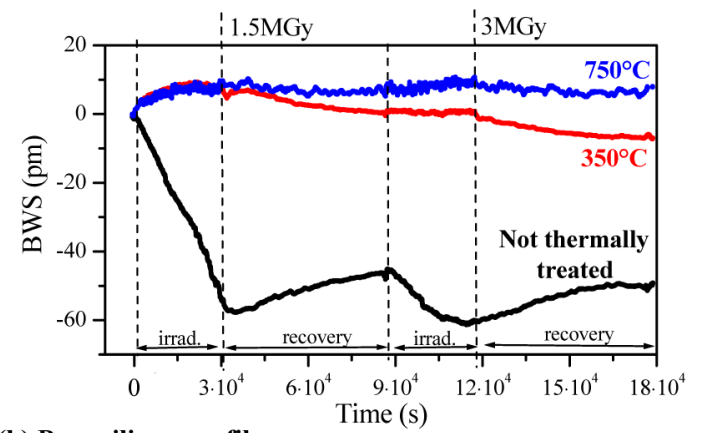

(b) Pure silica core fiber

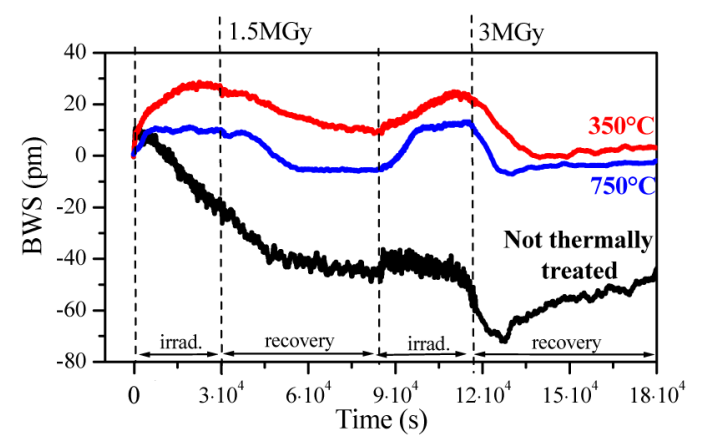

Fig. 3. Radiation-induced Bragg wavelength shift at RT, as a function of the time from the first irradiation start, for gratings written in (a) the F-doped core and (b) the PSC fibers and subjected to three different thermal treatments. The vertical dashed lines indicate the start and the end of the irradiation runs. In the upper part of each graph the total dose reached after a run is shown, dose-rate being $50 \mathrm{~Gy} / \mathrm{s}$.

the radiation resistance of the fs-FBGs: the higher the annealing temperature, the better the grating radiation resistance for our test conditions.

The radiation effect on the grating amplitude is below 5\%, as shown in Fig. $\underline{4}$ for the FBGs written in the F-doped core fiber. This means that our FBGs' amplitudes are nearly insensitive to the radiations. In details, the amplitude of the gratings not-treated or annealed at $350^{\circ} \mathrm{C}$ increases at the irradiation start, reaches a maximum, and then decreases; it continues decreasing during the recoveries. Whereas, for the grating annealed at $750^{\circ} \mathrm{C}$,

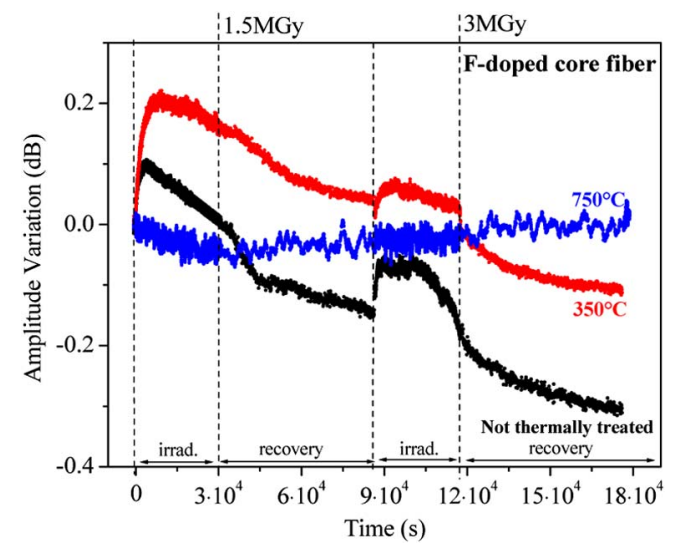

Fig. 4. Variation of the peak amplitude as a function of the time from the first irradiation start, for gratings written in the F-doped core fiber and subjected to three different thermal treatments (the dose-rate is $50 \mathrm{~Gy} / \mathrm{s}$ ). the global variation is smaller than $0.1 \mathrm{~dB}$ and it is not possible to observe a clear trend.

As a first conclusion, the FBGs written at laser power exceeding $500 \mathrm{~mW}$ and annealed at $750^{\circ} \mathrm{C}$ showed the lower radiation sensitivity at RT in comparison to the other gratings. For this reason, two other gratings (one inside each fiber type) pre-annealed at $750^{\circ} \mathrm{C}$ were irradiated with the same experimental setup, at a higher temperature of irradiation, $230^{\circ} \mathrm{C}$. To calculate the radiation-induced peak shift, reported in Fig. 5, the temperature effect on the BWS was compensated thanks to the sensitivity calculated around $230^{\circ} \mathrm{C}$ by varying the temperature in a range between $220^{\circ} \mathrm{C}$ and $240^{\circ} \mathrm{C}$. Especially at $230^{\circ} \mathrm{C}$, these preliminary measurements also allow us to ensure that the calculated BWS observed under irradiation after correction is only related to radiation effects. Indeed, if temperature affects the fixation system used for the FBG tests inducing some strain changes, these artefacts will be the same for both the preliminary and radiation run and will be compensated at the same time as the temperature drift of the FBG.

At this high temperature of irradiation, the BWS shows a saturating tendency. During the first run up to a 1.5 MGy dose, the peak of the grating written in the Fdoped fiber shifts toward longer wavelengths by $10 \mathrm{pm}$ [Fig. 5(a)], whereas that of the grating written in the PSC fiber shifts toward shorter wavelengths by $20 \mathrm{pm}$ [Fig. 5(b)]. However, independently of this first phase, during the recovery at high temperature, the peak shifts quickly toward shorter wavelengths of about $20 \mathrm{pm}$, whereas at the start of the second irradiation run it

(a) F-doped core fiber

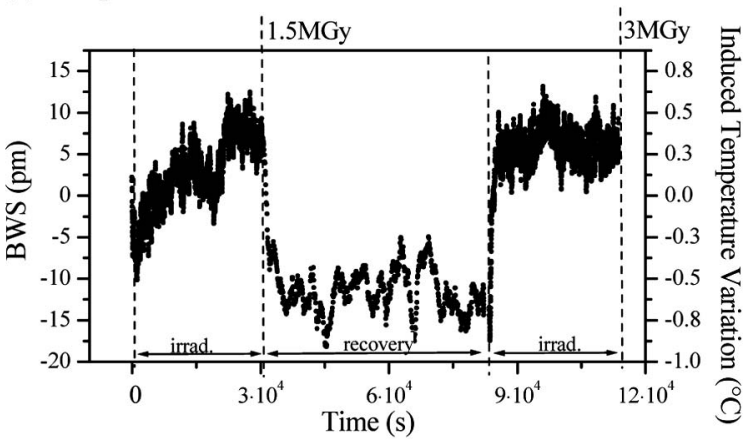

(b) Pure silica core fiber

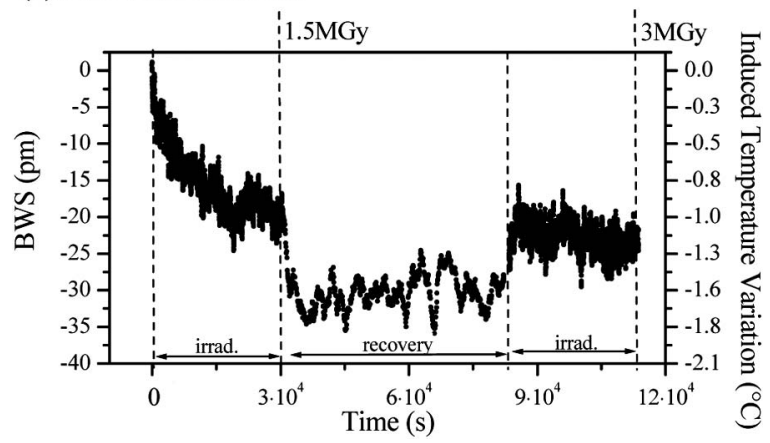

Fig. 5. Radiation-induced Bragg wavelength shift at about $230^{\circ}$ $\mathrm{C}$, as a function of the time from the first irradiation start, for gratings written in (a) the F-doped core and (b) the PSC fibers, initially pretreated at $750^{\circ} \mathrm{C}$. The $y$ axis on the right indicates the corresponding radiation-induced error when this component is used as a temperature sensor. 
red-shifts by the same quantity and it does not change anymore for a further dose of 1.5 MGy. The fast variation after the first run can be caused by the temperature held constant at $230^{\circ} \mathrm{C}$ during the recovery.

So, if the FBG is used as a temperature sensor in a radiative environment with a temperature between $20^{\circ} \mathrm{C}$ and $230^{\circ} \mathrm{C}$ and an accumulated dose of $3 \mathrm{MGy}$, the error on the temperature measurement because of the radiation is lower than $1.5^{\circ} \mathrm{C}$, for the gratings written in both F-doped and PSC fibers and annealed at $750^{\circ} \mathrm{C}$.

In literature, only very few works $[10,11]$ have been realized about radiation effects on FBG written with femtosecond laser light. The observed radiation-induced BWS was small with respect to the gratings written with UV light (within $10 \mathrm{pm}$ for the F-doped or PSC fibers, at an accumulated dose below 1 MGy), but no saturating behavior had been observed until now. Moreover, for the first time, we demonstrated the thermal pretreatment efficiency to increase the radiation resistance of the fsFBG in radiation tolerant fibers. The higher the annealing temperature, the smaller the radiation induced BWS; whereas the amplitude of these FBGs is nearly radiation insensitive. This result, indeed, is in contradiction to those reported in literature [11,12], according to which the higher the temperature of the pre-irradiation treatment, the higher the grating radiation sensitivity, independently of the writing light (cw UV laser or IR fs-one). According to these authors, some centers generated during the grating inscription recombine during the annealing, restoring the precursors. In our case, the BWS has been clearly reduced by the thermal pretreatment and this effect should be explained by a concentration reduction not only of defects but also of precursors. However, this should be verified. The same positive effect on the BWS was observed for a pre-irradiation.

In summary, we have studied fiber Bragg gratings in radiation-resistant fibers, as F-doped ones. These FBGs are written with femtosecond radiation at $800 \mathrm{~nm}$ and are exposed to a thermal annealing pretreatment at $750^{\circ} \mathrm{C}$ to enhance both their thermal stability and their radiation tolerance. When the irradiation up to $3 \mathrm{MGy}$ is performed at a temperature between $25^{\circ} \mathrm{C}$ and $230^{\circ} \mathrm{C}$, on the basis of measured BWS the temperature error is about $1.5^{\circ} \mathrm{C}$ and $1.0^{\circ} \mathrm{C}$ for the gratings written in the PSC and in the F-doped fiber, respectively.

These results are relevant because they allow us to design temperature sensors based on these FBGs for the nuclear industry that can survive the harsh environment encountered in accidental conditions. Studies are in progress to evaluate the potential of these radiation tolerant FBGs at higher doses and temperatures such as the ones expected around the cores of the next generation of nuclear power plants mixing neutrons and $\gamma$ rays.

\section{References}

1. IAEA-TECDOC-1188, "Assessment and management of ageing of major nuclear power plant components important to safety: in-containment instrumentation and control cables."

2. A. I. Gusarov and S. K. Hoeffgen, IEEE Trans. Nucl. Sci. 60, 2037 (2013).

3. S. Girard, J. Kuhnhenn, A. Gusarov, B. Brichard, M. Van Uffelen, Y. Ouerdane, A. Boukenter, and C. Marcandella, IEEE Trans. Nucl. Sci. 60, 2015 (2013).

4. H. Henschel, S. K. Hoeffgen, K. Krebber, J. Kuhnhenn, and U. Weinand, IEEE Trans. Nucl. Sci. 55, 2235 (2008).

5. H. Henschel, S. K. Hoeffgen, J. Kuhnhenn, and U. Weinand, IEEE Trans. Nucl. Sci. 57, 2029 (2010).

6. K. O. Hill and G. Meltz, J. Lightwave Technol. 15, 1263 (1997).

7. S. J. Mihailov, D. Grobnic, C. W. Smelser, P. Lu, R. B. Walker, and H. Ding, Laser Chem. 2008, 416251 (2008).

8. C. W. Smelser, S. J. Mihailov, and D. Grobnic, Opt. Lett. 29, 2127 (2004).

9. S. J. Mihailov, C. W. Smelser, D. Grobnic, R. B. Walker, P. Lu, H. Ding, and J. Unruh, J. Lightwave Technol. 22, 94 (2004).

10. D. Grobnic, H. Henschel, S. K. Hoeffgen, J. Kuhnhenn, S. J. Mihailov, and U. Weinand, Proc. SPIE 7316, 73160C (2009).

11. H. Henschel, D. Grobnic, S. K. Hoeffgen, J. Kuhnhenn, S. J. Mihailov, and U. Weinand, IEEE Trans. Nucl. Sci. 58, 2103 (2011).

12. A. Gusarov, S. Vasiliev, O. Medvedkov, I. Mckenzie, and F. Berghmans, IEEE Trans. Nucl. Sci. 55, 2205 (2008). 